\title{
Overexpression of MARCKS indicates a poor prognosis of oral squamous cell carcinoma
}

\author{
CHENGJING LI ${ }^{1}$, RONG XIA ${ }^{1}$, HAOWEI XUE ${ }^{2}$, YUKUN HU ${ }^{2}$, MING SUN $^{2}$, \\ DONGDONG FANG ${ }^{1}$, WENYU YANG ${ }^{1}$, FENG XIAO $^{1}$ and JUN HOU ${ }^{1,2}$ \\ ${ }^{1}$ Department of Oral and Maxillofacial Surgery, The Second Affiliated Hospital of Anhui Medical University; \\ ${ }^{2}$ Department of Oral and Maxillofacial Surgery, The First Affiliated Hospital of Anhui Medical University, \\ Hefei, Anhui 230001, P.R. China
}

Received December 4, 2017; Accepted July 10, 2018

DOI: $10.3892 / \mathrm{ol} .2018 .9311$

\begin{abstract}
Myristoylated alanine-rich $\mathrm{C}$ kinase substrate (MARCKS) is a protein kinase $\mathrm{C}$ substrate functioning in different physiological and pathological mechanisms. Previous studies have suggested that MARCKS is capable of influencing tumorigenesis and progression. However, a limited number of studies are available regarding the role of MARCKS in oral squamous cell carcinoma (OSCC). The present study primarily examined MARCKS expression in the OSCC tissues. Furthermore, increased expression of MARCKS was confirmed in the majority of OSCC tissues. Increased MARCKS expression was correlated with more advanced tumor stages, lymphatic metastasis and a poorer overall patient survival. Further molecular mechanistic examinations revealed that downregulated MARCKS expression inhibited the proliferation and migration of OSCC cells in vitro through interruption of MARCKS expression. In addition, the present study demonstrated that MARCKS aggravated OSCC progression via the phosphoinositide 3-kinase/protein kinase $\mathrm{B}$ pathway. Accordingly, the present study considered MARCKS to be a promoter of OSCC tumorigenesis and progression, with the potential utility as a biomarker of a poor prognosis.
\end{abstract}

\section{Introduction}

Oral cancer is a common malignant tumor observed in the neck and the head area $(1,2)$. Oral squamous cell carcinoma (OSCC) is among the most frequent pathological types of oral cancer $(3,4)$. Despite recent breakthroughs in surgical, chemoradiotherapeutic and biological treatments, as well as molecular targeting therapy, the long-term therapeutic impact remains

Correspondence to: Professor Jun Hou, Department of Oral and Maxillofacial Surgery, The First Affiliated Hospital of Anhui Medical University, 218 Jixi Road, Hefei, Anhui 230001, P.R. China E-mail: houjun163163@126.com

Key words: myristoylated alanine-rich $\mathrm{C}$ kinase substrate, oral squamous cell carcinoma, metastasis, proliferation, prognosis poor $(5,6)$. Additionally, the clinical signs of $\sim 2 / 3$ patients with OSCC in the early stages are not distinguishable as the disease is typically diagnosed in the advanced stages (7). The early diagnosis and treatment of OSCC serve substantial roles in the improvement of the prognosis and survival rate of the disease (8). Accordingly, the detection of new molecular markers is suspected to be vital in the improvement of the prognosis of OSCC.

Myristoylated alanine-rich C kinase substrate (MARCKS) is regarded as a renowned specific substrate of protein kinase C, extensively observed among different human tissues (9). MARCKS is known to participate in cell motility, phagocytosis and membrane trafficking, as well as mitogenesis (10). Notably, previous studies have shed light on the likely involvement of MARCKS in the tumorigenesis and progression of different malignancies, including brain cancer (11), thyroid cancer (12), colon cancer (13), melanoma (14), glioblastoma cancer (15) and cholangiocarcinoma (16). However, relatively few studies are available regarding the role of MARCKS in the proliferation of OSCC.

In the present study, the levels of MARCKS protein and mRNA were investigated in the OSCC tissue using western blotting and reverse transcription-quantitative polymerase chain reaction (RT-qPCR). The present study also evaluated the association between the expression of MARCKS and the prognosis of patients with OSCC. Additionally, the potential molecular mechanism of MARCKS promoting the progression of OSCC was also further analyzed.

\section{Materials and methods}

Tissue specimens. In the present study, OSCC tissues were immersed in $4 \%$ formalin for $4 \mathrm{~h}$ at room temperature and then were embedded in paraffin. Subsequently, the tissue specimens were cut into sections with a thickness of $4 \mu \mathrm{m}$ and obtained from 128 patients with OSCC (78 males and 50 females; Age range: 24-76 years old; Median age, 41 years). The tumor tissues and the corresponding normal peri-tumor samples were obtained from patients who had undergone surgical resection at the First and Second Affiliated Hospitals of Anhui Medical University (Hefei, China) between June 2009 and December 2012. The peri-tumor samples were used 
as negative controls in the present study. Written informed consent was obtained from each patient for the use of their tissue specimens in the present study. Additionally, the present study was approved by the Anhui Medical University Ethical Review Board, according to the Declaration of Helsinki. None of the patients had undergone any type of preoperative treatment prior to the curative surgery. Independent classification of the pathological stage and tumor grade (including poorly-, moderately- and well-differentiated) was performed by two pathologists in accordance with the American Joint Committee on Cancer Tumor-Node-Metastasis (TNM) staging system.

Immunohistochemistry. The MARCKS antibody (catalog no., ab52616; Abcam, Cambridge, MA, USA) was used to detect MARCKS expression. All sections were gradually deparaffinized and rehydrated with xylene (twice for $10 \mathrm{~min}$ each time) and ethanol (100\%, twice, for 5 min each; $95 \%$, $5 \mathrm{~min}$; 90\%, $5 \mathrm{~min}$; 90\%, $5 \mathrm{~min}$; 85\%, $5 \mathrm{~min}$; 75\%, $5 \mathrm{~min}$ ) at room temperature. Antigen retrieval was performed by heating the sections in $10 \mathrm{mM}$ sodium urinary citrate at $95^{\circ} \mathrm{C}$ for $\sim 30 \mathrm{~min}$. Additionally, $0.3 \%$ hydrogen peroxide was applied to block endogenous peroxidase activity for $10 \mathrm{~min}$ at room temperature. According to the manufacturer's protocol, the MARCKS antibody was diluted at 1:200 and was added dropwise to the sections, which were subsequently incubated overnight at $4^{\circ} \mathrm{C}$. Subsequently, the avidin-biotin-peroxidase complex (Shanghai Guge Biotechnology Co., Ltd, Shanghai, China) was used for staining. PBS or normal mouse serum was applied as a negative control. All sections were subsequently counterstained with hematoxylin for $30 \mathrm{sec}$ at room temperature. Next, images of each of the sections were captured. MARCKS expression was scored using a semi-quantitatively immunostaining score system that was subsequently used in the evaluation of the intensity of staining and the percentage of stained cells. Additionally, the percentage score of stained cells represents the proportion of positive cells: $5,>75 \%$ of cells stained; $4,50-75 \% ; 3,10-50 \%$; $2,5-10 \% ; 1,<5 \%$. The staining intensities of stained cells were scored as follows: 0, no staining; 1 , weak positive staining; 2, moderate positive staining; and 3, strong positive staining. Subsequently, the overall score was calculated by multiplying the percentage score by the intensity score (0-15). The expression level of MARCKS (high or low) was determined by the total score as follows: high expression, $\geq 7$; and low expression, $<7$. Independent investigations of all these scores were performed by two researchers and the average score was statistically analyzed. Subsequently, the OLYMPUS light microscope (BX41; x200 magnification) was used to capture immunohistochemical images.

Cell culture and transfection. Two human oral squamous cell carcinoma cell lines, Cal27 and HN12, were cultured in Dulbecco's modified Eagle medium, containing $10 \%$ fetal bovine serum (FBS), $1 \%$ penicillin and streptomycin, in a dampened atmosphere of $5 \% \mathrm{CO}_{2}$ at $37^{\circ} \mathrm{C}$. RNA interference short interfering RNAs (siRNAs) were obtained from RuiBo Biology Co., Ltd. (Guangzhou, China). Two siRNAs (siRNA1, 5'-CTACACTTGGGCTCCTTTT-3'; and siRNA2, 5'-GGUGCCCAGUUCUCCAAGAUU-3') were used to reduce the expression of MARCKS. However, OSCC Cal27 and HN12 cells transfected with siRNA1 exhibited higher knockdown efficiency than those transfected with siRNA2 (data not shown). The empty control cells were transfected with non-targeting MARCKS siRNA (5'-CGCACCAGAACA AACACAUU-3'). In order to deliver the targeted siRNA, Cal27 and HN12 cells were incubated at 30-50\% confluency, followed by the addition of $20 \mathrm{nM}$ siRNAs supplemented with $5 \mu$ RNAiMAX (Invitrogen; Thermo Fisher Scientific, Inc., Waltham, MA, USA).

$R T$ - $q P C R$. Total RNA was extracted from the cell lines using TRIzol (Invitrogen; Thermo Fisher Scientific, Inc.), according to the manufacturer's protocol. The absorbance of RNA was measured at $260 \mathrm{~nm}$ using a NanoDrop spectrophotometer (ND-1000; Thermo Fisher Scientific, Inc.), in order to determine the total RNA concentration. Reverse transcription of $2 \mu \mathrm{g}$ total RNA was conducted using the Prime Script RT reagent kit, gDNA Eraser (Takara Bio, Inc., Otsu, Japan). According to the manufacturer's protocol, the ABI 7500 fast real-time PCR system (Applied Biosystems; Thermo Fisher Scientific, Inc.) and the SYBRGreen PCR Master mix (Applied Biosystems; Thermo Fisher Scientific, Inc.) were used with a first step at $95^{\circ} \mathrm{C}$ for $10 \mathrm{~min}$, followed by 40 cycles with $95^{\circ} \mathrm{C}$ for $15 \mathrm{sec}$ and $60^{\circ} \mathrm{C}$ for $1 \mathrm{~min}$, with a fluorescent reading at the end of this step to amplify the specific genes. The primers were as follows: MARCKS forward, 5'-AGCCCGGTAGAGAAGGAGG-3' and reverse, 5'-TTGGGCGAAGAAGTCGAGGA-3'; and GAPDH forward, 5'-CGTCCCGTAGACAAAATGGT-3' and reverse, 5'-TTGATGGCAACAATCTCCAC-3'. GAPDH was used as the interval control to calculate the relative expression level of MARCKS using the comparative delta Cq $\left(2^{-\Delta \Delta \mathrm{Cq}}\right)$ method (17). Furthermore, independent determination of each sample was performed thrice, and the mean value of the expression levels was calculated.

Transwell assay. Transwell cell migration assays were performed in 24-well plates with $8.0-\mu \mathrm{m}$ permeable polycarbonate membranes. Matrigel inserts were used (BD Biosciences, San Diego, CA, USA). Cells were subsequently diluted with serum-free basic culture DMEM (Gibco; Thermo Fisher Scientific, Inc.) at $5 \times 10^{5}$ cells $/ \mathrm{ml}$, followed by transferring 0.5-1 $\mathrm{ml}$ to each Transwell using pipette guns. Additionally, culture medium with $10 \%$ FBS was inserted into the lower chamber wells. Subsequently, the wells were incubated at a temperature of $37^{\circ} \mathrm{C}$ in a moistened cell culture incubator for $24 \mathrm{~h}$. The non-invading cells on the upper side of the membrane were removed by scrubbing. The invading cells were fixed with $10 \%$ formalin for $10 \mathrm{~min}$, followed by staining with $0.1 \%$ crystal violet for $20 \mathrm{~min}$ at room temperature. The OLYMPUS light microscope (BX41; x200 magnification) was used to capture images.

Cell colony formation assay. Cells in the logarithmic phase were diluted to $1-1.5 \times 10^{3}$ cells $/ \mathrm{ml}$ by $10 \%$ FBS culture medium, followed by drop by drop addition of $1 \mathrm{ml}$ to each well of 6-well plates supplemented with $1 \mathrm{ml}$ culture medium. Subsequently, the cells were incubated at a temperature of $37^{\circ} \mathrm{C}$ in a moistened cell culture incubator for 2 weeks. The 

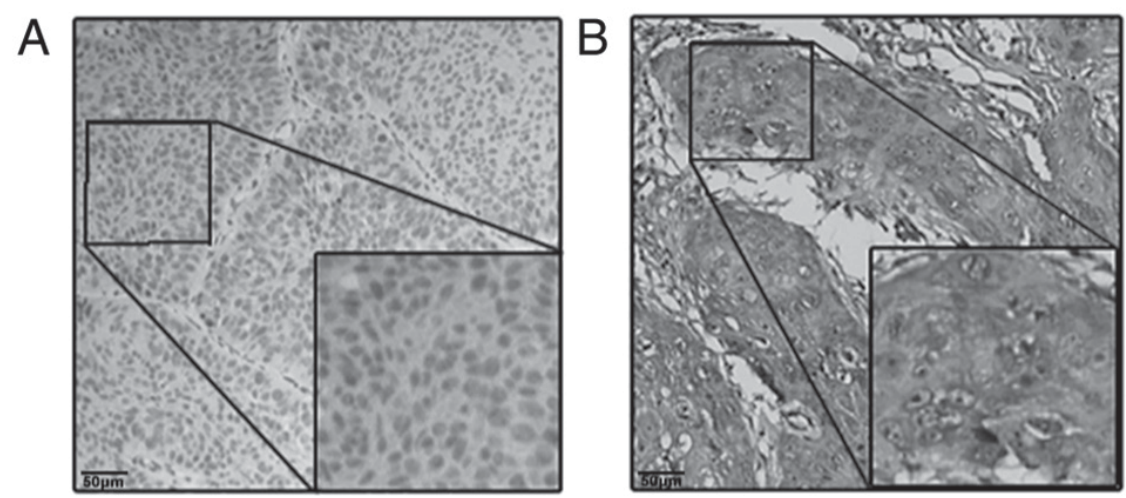

Figure 1. MARCKS protein expression was detected using immunohischemistry in OSCC tissue samples. (A) High and (B) low MARCKS expression in OSCC tissues. Scale bar=50 $\mu \mathrm{m}$. MARCKS, myristoylated alanine-rich C kinase substrate; OSCC, oral squamous cell carcinoma.

culture medium was replaced with new medium. Following washing with PBS twice in 2 weeks, the cells were fixed with absolute methanol for 15 mins, and staining with Giemsa stock solution for $10 \mathrm{mins}$ at room temperature.

Western blotting. Radioimmunoprecipitation assay (Beyotime Institute of Biotechnology, Haimen, China) for the purpose of lysing the accumulated cells and the protease inhibitor cocktail (Roche Diagnostics, Basel, Switzerland) was added in order to prevent denaturation. The cells were incubated on ice for $30 \mathrm{~min}$. Next, the samples were centrifuged at $12,000 \mathrm{x}$ g at $4^{\circ} \mathrm{C}$ for $\sim 30 \mathrm{~min}$. Subsequently, the total protein concentration was determined using a bicinchoninic acid protein assay kit (Beyotime Institute of Biotechnology). In order to break the protein structure, the protein was heated at $100^{\circ} \mathrm{C}$ for $10 \mathrm{~min}$. Subsequently, equal amounts ( $40 \mu \mathrm{g}$ ) of protein were added to each well of $10 \%$ polyacrylamide gels. Following electrophoresis, the protein was transferred to nitrocellulose membranes, followed by blocking in 5\% bovine serum albumin or 5\% skimmed milk diluted in Tris-buffered saline Tween (TBST) for $2 \mathrm{~h}$ at room temperature. Next, the blocked membrane was incubated with the following primary antibodies: MARCKS (dilution, 1:1,000; catalog no., 5607; Cell Signalling Technology, Inc., Danvers, MA, USA), phosphoinositide 3-kinase (PI3K; dilution, 1:1,000; catalog no., 4292; Cell Signalling Technology, Inc.), protein kinase B (Akt; dilution, 1:1,000; catalog no., 9272; Cell Signalling Technology, Inc.), phosphorylated PI3K (p-PI3K; dilution, 1:1,000; catalog no., 13857; Cell Signalling Technology, Inc.), p-Akt (dilution, 1:1,000; catalog no., 9611; Cell Signalling Technology, Inc.) and $\beta$-actin (dilution, 1:1,000; catalog no., M20011; Abmart Co., Ltd., Shanghai, China) at $4^{\circ} \mathrm{C}$ overnight. Subsequent to washing the membranes with TBST, the membranes were incubated with a horseradish peroxidase-conjugated donkey anti-goat secondary antibody (dilution, 1:5,000; catalog no., KC-RB-035; Zhejiang Kangchen Biotech Co., Ltd., Wuhan, China) for $\sim 60 \mathrm{~min}$ at room temperature. Following washing of the membrane with TBST, the membranes were visualized using an enhanced chemiluminescence kit (Thermo Fisher Scientific, Inc.), followed by capturing the emitted signals using KODAK X-OMAT BT Film (Kodak, Rochester, NY, USA). The gray value of the protein was measured by employing ImageJ software (National Institutes of Health, Bethesda, MD, USA).



Figure 2. MARCKS mRNA levels in OSCC specimens were determined by reverse transcription-quantitative polymerase chain reaction. MARCKS mRNA was significantly higher in OSCC tissues than in their paired peri-tumor counterparts. ${ }^{*} \mathrm{P}<0.05$ vs. peri-tumor group. MARCKS, myristoylated alanine-rich $\mathrm{C}$ kinase substrate; OSCC, oral squamous cell carcinoma.

Statistical analysis. SPSS statistical software (version 13.0; SPSS, Inc., Chicago, IL, USA) was used to analyze the immunohistochemical and clinicopathological data. Data are presented as the mean \pm standard deviation of at least three independent experiments. Student's t-tests were used to compare the levels of MARCKS mRNA in tumor and corresponding peri-tumor samples. Additionally, $\chi^{2}$ was used to compare immunohistochemistry results and clinicopathological factors. The analysis of the survival curves was performed using the Kaplan-Meier method. The long-rank test was used for the determination of the discrepancy between different groups. Furthermore, the Cox proportional hazards framework was used to analyze the survival differences among various groups. $\mathrm{P}<0.05$ was considered to indicate a statistically significant difference.

\section{Results}

Abnormal expression of MARCKS in OSCC tissues. In order to investigate the function of MARCKS in the advancement of OSCC, the evaluation of the MARCKS mRNA and protein expression levels was performed in the OSCC and the corresponding normal tissues. The results of the present study suggested that the higher protein expression level of MARCKS 
Table I. MARCKS expression in relation to clinical and pathological factors in 128 patients with OSCC.

\begin{tabular}{|c|c|c|c|c|}
\hline \multirow[b]{2}{*}{ Variables } & \multicolumn{2}{|c|}{$\begin{array}{l}\text { MARCKS } \\
\text { expression }\end{array}$} & \multirow[b]{2}{*}{$\chi^{2}$} & \multirow[b]{2}{*}{ P-value } \\
\hline & $\begin{array}{l}\text { Low } \\
(n=41)\end{array}$ & $\begin{array}{l}\text { High } \\
(\mathrm{n}=87)\end{array}$ & & \\
\hline Sex & & & 1.371 & 0.242 \\
\hline Male & 28 & 50 & & \\
\hline Female & 13 & 37 & & \\
\hline Age (years) & & & 1.053 & 0.305 \\
\hline$\leq 60$ & 24 & 59 & & \\
\hline$>60$ & 17 & 28 & & \\
\hline Size $(\mathrm{cm})$ & & & 0.012 & 0.913 \\
\hline$\leq 4$ & 31 & 65 & & \\
\hline$>4$ & 10 & 22 & & \\
\hline Smoking and drinking & & & 0.001 & 0.969 \\
\hline None & 19 & 40 & & \\
\hline Yes & 22 & 47 & & \\
\hline Differentiation & & & 0.230 & 0.631 \\
\hline Well & 28 & 63 & & \\
\hline Moderate and poor & 13 & 24 & & \\
\hline $\mathrm{T}$ classification & & & 2.074 & 0.150 \\
\hline $\mathrm{T} 1+\mathrm{T} 2$ & 29 & 50 & & \\
\hline $\mathrm{T} 3+\mathrm{T} 4$ & 12 & 37 & & \\
\hline $\mathrm{N}$ classification & & & 7.420 & $0.006^{\mathrm{a}}$ \\
\hline No & 32 & 46 & & \\
\hline $\mathrm{N} 1-3$ & 9 & 41 & & \\
\hline TNM stage & & & 4.243 & $0.039^{\mathrm{a}}$ \\
\hline I-II & 23 & 32 & & \\
\hline III-IV & 18 & 55 & & \\
\hline
\end{tabular}

TNM, tumor-node metastasis. ${ }^{\mathrm{a}} \mathrm{P}<0.05$.

was observed in the cytoplasm of tumor cells in 87 cases $(67.9 \%)$ and in the cytoplasm of matched peri-tumor normal cells in 21 cases $(16.4 \%)$, a difference that was statistically significant $(\mathrm{P}<0.001$; Fig. 1). The same results were observed in the assessment of MARCKS mRNA in 27 patients with OSCC using RT-qPCR (Fig. 2).

MARCKS is associated with lymphatic metastasis and a poor prognosis of OSCC. Table I. shows that $\chi^{2}$ test analysis revealed that the expression of MARCKS was higher in the advanced-grade tumors $(\mathrm{P}<0.05)$, and that high expression of MARCKS was correlated with lymphatic metastasis $(\mathrm{P}<0.05)$. In summary, as suggested by the results of the present study, MARCKS may have substantial impact on the metastasis or invasion of OSCC.

For further assessment of the use of MARCKS expression in the prognosis of patients with OSCC, retrospective analysis of the follow-up data of 128 cases was performed. Fig. 3 demonstrates that the OSCC patients with higher MARCKS expression



Figure 3. Kaplan-Meier curves for differential expression of MARCKS in oral squamous cell carcinoma. Patients with high levels of MARCKS expression had a poor overall survival $(\mathrm{P}=0.001)$. MARCKS, myristoylated alanine-rich $\mathrm{C}$ kinase substrate.

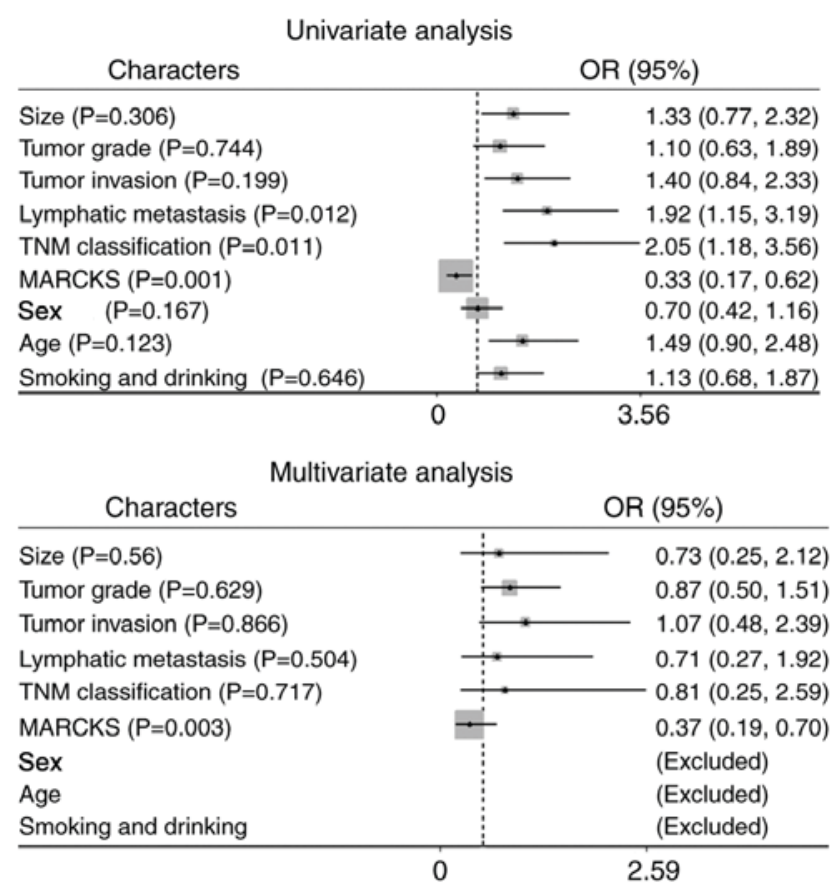

Figure 4. Univariate and multivariate analysis of prognostic factors for overall survival in patients with oral squamous cell carcinoma. The results are presented as forest plots. OR, odds ratio.

exhibited improved overall survival (OS), as determined by Kaplan-Meier analysis $(\mathrm{P}<0.05)$. In addition, univariate analysis demonstrated that the high MARCKS expression level was an independent prognostic factor for a poor OS (Fig. 4A, HR=0.33, 95\% $\mathrm{CI}=0.17-0.62, \mathrm{P}=0.001$ ), along with $\mathrm{TNM}$ stage and lymphatic metastasis $(\mathrm{HR}=2.05,95 \% \mathrm{CI}=1.18-3.56, \mathrm{P}=0.011$; $\mathrm{HR}=1.92,95 \% \mathrm{CI}=1.15-3.19, \mathrm{P}=0.012$, respectively). Multivariate Cox regression analysis further revealed the prognostic value of MARCKS (Fig. 4B, HR=0.37, 95\% CI=0.19-0.70, $\mathrm{P}=0.003$ ). In brief, the results of the present study demonstrated that the 


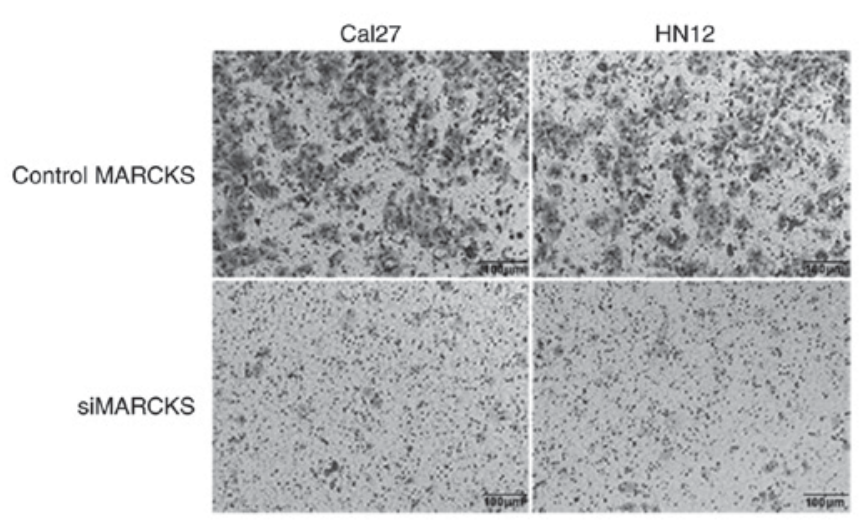

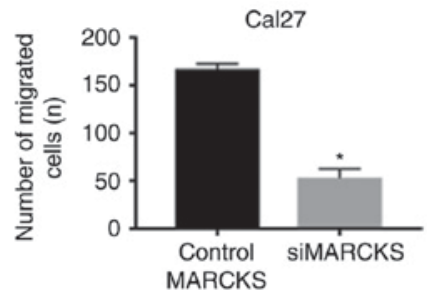

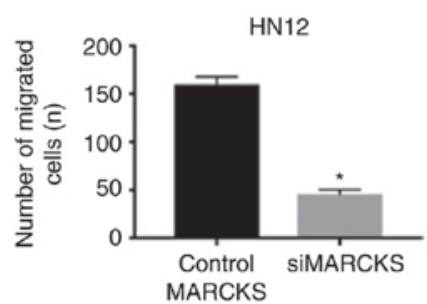

Figure 5. Cell migratory ability detected by Transwell migration assay. Representative image and statistical analysis results are presented in the left and right panels, respectively. ${ }^{*} \mathrm{P}<0.05$ vs. the control group. siMARCKS.
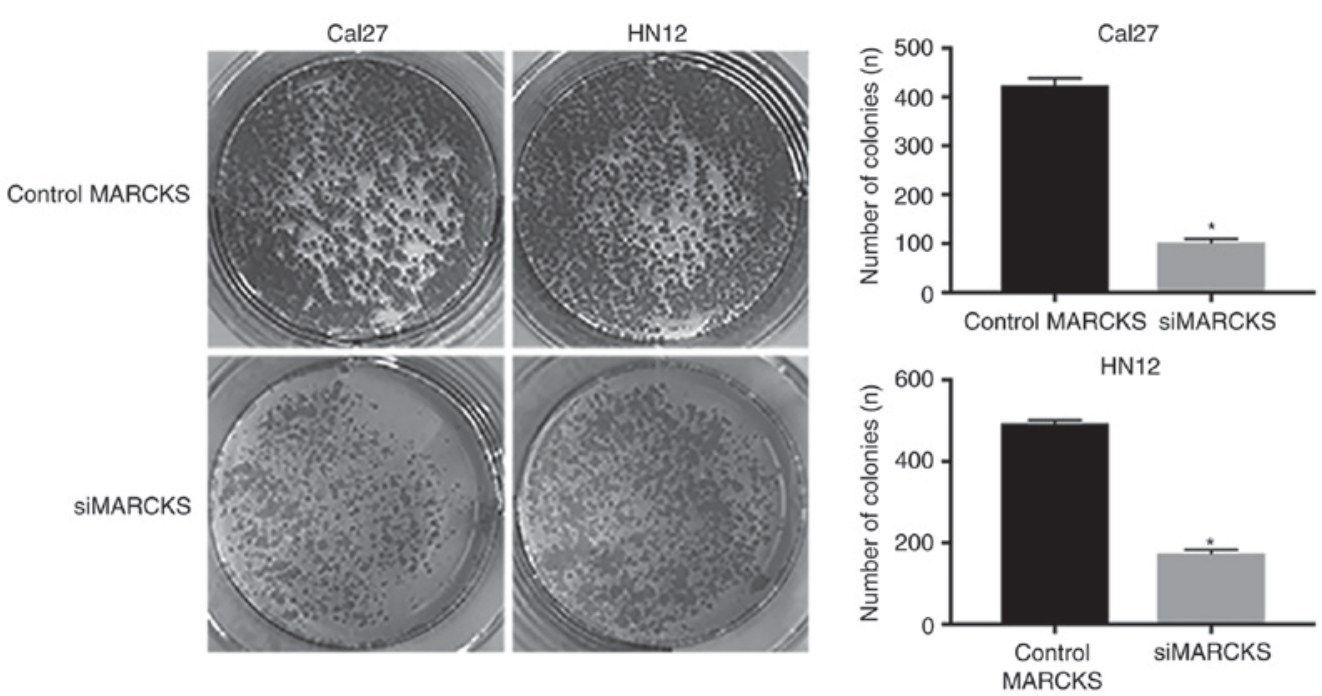

Figure 6. Cell proliferation ability detected by colony formation assay. Representative image and statistical analysis results are presented in the left and right panels, respectively. " $\mathrm{P}<0.05$ vs. the control group. siMARCKS, myristoylated alanine-rich $\mathrm{C}$ kinase substrate short interfering RNA.

expression pattern of MARCKS in OSCC can be applied as an independent risk factor for the prognosis prediction of the disease.

Inhibiting the expression of MARCKS decreases the mobility and proliferation of OSCC cells. In order to investigate the fundamentalmolecularmechanisms of theMARCKS-mediated progression of OSCC, siRNA was used to decrease the MARCKS expression in the OSCC Cal27 and HN12 cell lines. Transwell migration and invasion assays suggested that the downregulated expression level of MARCKS expressively inhibited the tumor cell mobility compared with that of the control cells (Fig. 5). Furthermore, the colony formation assay was employed to identify the impacts of MARCKS expression on the OSCC cell proliferation. A notable difference was observed in the tumor cell proliferation between the control group and the MARCKS-knockdown group in the Cal27 and HN12 cells (Fig. 6). Taken together, these data indicated that MARCKS contributes toward the mobility and proliferation of OSCC cells.
MARCKS may regulate the progression of OSCC through the PI3K/Akt pathway. It has been demonstrated that MARCKS is involved in regulating the PIP3 located upstream of the PI3K/Akt pathway. Accordingly, we hypothesized that MARCKS regulates the progression of OSCC through the PI3K/Akt pathway. Western blot analysis was employed to identify the changes in p-PI3K and p-Akt in the OSCC cell lines following interruption of MARCKS. Fig. 7 demonstrates that PI3K and Akt phosphorylation were decreased while decreasing MARCKS expression in the tumor cell lines. This result suggested that the PI3K/Akt pathway was involved in the MARCKS-mediated progression of OSCC.

\section{Discussion}

MARCKS is a protein kinase $\mathrm{C}$ substrate that is implicated in the adhesion, secretion and motility of cells using the regulation of the actin cytoskeletal structure (18). MARCKS protein was primarily identified in the rat brain synaptosomes by Ueno et al (19). A previous study reported that MARCKS 

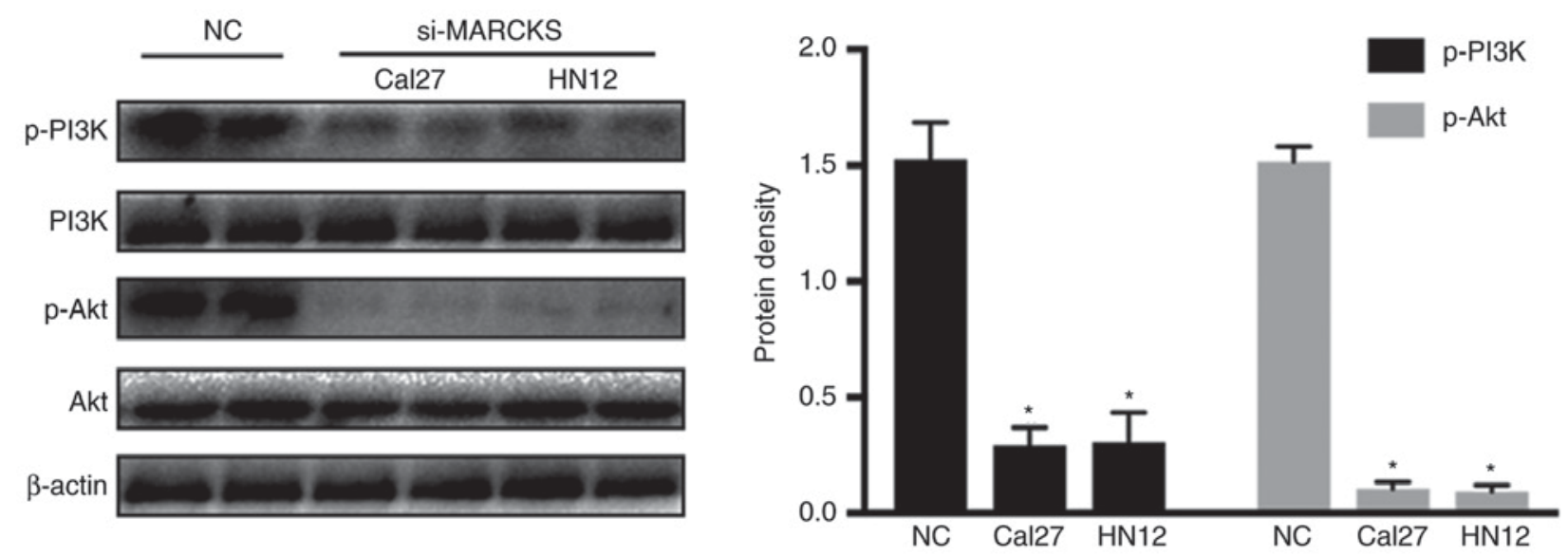

Figure 7. Western blot analysis indicated that MARCKS mediated the oral squamous cell carcinoma progression via the PI3K/Akt pathway. The gray values of protein are presented in the right panel. ${ }^{~} \mathrm{P}<0.05$ vs. the $\mathrm{NC}$ group. $\mathrm{NC}$, negative control; siMARCKS, myristoylated alanine-rich $\mathrm{C}$ kinase substrate short interfering RNA; p-PI3K, phosphorylated phosphoinositide 3-kinase; p-Akt, phosphorylated protein kinase B.

serves a pivotal role in different malignant tumors (20). Previous studies revealed that abnormal MARCKS expression is associated with the diagnosis and prognosis of patients with cancer $(21,22)$. Manai et al $(21)$ reported MARCKS-overexpression in inflammatory breast cancer, and that this was associated with a poor metastasis-free survival. Hanada et al (22) also concluded that MARCKS could serve as a potential biomarker for the human primary lung squamous cell carcinoma using high-throughput screening methodology. Furthermore, drug resistance of tumors could be regulated by MARCKS expression. Chen et al (23) suggested that knockdown of MARCKS decreased the IC50 of regorafenib in the renal cancer cells. Taken together, these results suggested that MARCKS participates in the malignant biological properties and may serve as a promising therapeutic target.

ThePI3K/Aktsignallingpathwaywasinvolvedincellproliferation, survival, motility and metabolism, tumorigenesis, and tumor progression (24-26). Phosphatidylinositol-4,5-bisphosphate (PIP2), together with phosphatidylinositol-4 and 5-bisphosphate (PIP3), functions as a pivotal link in the PI3K/Akt signalling pathway. A recent study demonstrated that MARCKS is persistently hyperphosphorylated by either a PKC-dependent and/or a PKC-independent manner, resulting in its cytosolic retention, as well as the accessibility of PIP2 to PI3K (27). This further progress increases Akt activation, giving rise to tumor cell proliferation and acquired resistance. The present study developed the preliminary investigation of the effects of MARCKS on OSCC progression.

The present study revealed high MARCKS expression in OSCC tumor specimens for the purpose of being positively associated with the advanced tumor stage and lymph node metastasis. Additionally, an association was observed between high MARCKS expression and a decreased overall survival rate in patients with OSCC. Univariate and multivariate regression analyses supported the value of MARCKS as an independent risk prognostic factor of OSCC. Furthermore, the present study investigated the impact of MARCKS expression on the metastasis and proliferation of OSCC cells through the use of siRNA interruption methodology. A substantially decreased growth rate in the Cal27-si-MARCKS and HN12-si-MARCKS cells was determined by comparing it with that of the respective control cells. In addition, the present study determined apparent variations in tumor cell migration and infiltrations between the MARCKS inhibiting and control cells. These results supported the hypothesis that upregulated MARCKS enhances the malignant phenotype. With regards to the role of MARCKS in cancer, the available mechanistic studies have drawn attention to the Akt pathway. In this way, the present study also determined the associated between the protein expression level of MARCKS, and the phosphorylation of PI3K and Akt using western blot analysis.

The results of the present study suggested that MARCKS may be an independent prognosis factor for patients with OSCC. MARCKS aids in improving tumor cell invasion and metastasis via the PI3K/Akt pathway. However, more comprehensive investigations are required for unravelling the mechanism through which MARCKS regulates the tumorigenesis and progression of OSCC.

\section{Acknowledgements}

Not applicable.

\section{Funding}

The present study was supported by the Anhui Technological Research for Public Welfare Project Funding (grant no. 1704f0804023).

\section{Availability of data and materials}

The datasets used and/or analyzed during the present study are available from the corresponding author on reasonable request.

\section{Authors' contributions}

CJ performed the majority of the study and was a major contributor in writing the manuscript. CJ, RX, HW, YK and JZ performed the experiment. MS, DD, WJ and FX collected 
and analyzed the clinical data. $\mathrm{CJ}$ and $\mathrm{JH}$ made substantial contributions to the design of the work, drafting the manuscript and revising it critically for important intellectual content. All authors gave final approval of the version to be published.

\section{Ethics approval and consent to participate}

All human samples were collected with patients' written informed consent and approval from the Institute Research Medical Ethics Committee of Anhui Medical University (Hefei, China).

\section{Patient consent for publication}

Written informed consent was obtained from all subjects for the publication of their data.

\section{Competing interests}

The authors declare that they have no competing interests.

\section{References}

1. Kademani D: Oral cancer. Mayo Clin Proc 82: 878-887, 2007.

2. Ong TK, Murphy C, Smith AB, Kanatas AN and Mitchell DA Survival after surgery for oral cancer: A 30-year experience. Br J Oral Maxillofac Surg 55: 911-916, 2017.

3. Montero PH and Patel SG: Cancer of the oral cavity. Surg Oncol Clin N Am 24: 491-508, 2015.

4. Saeed NR and Gold JA: Oral squamous cell carcinoma. BMJ 308: 1372-1373, 1994

5. Wang ZQ, Liu K, Huo ZJ, Li XC, Wang M, Liu P, Pang B and Wang SJ: A cell-targeted chemotherapeutic nanomedicine strategy for oral squamous cell carcinoma therapy. J Nanobiotechnology 13: 63, 2015.

6. Xi S and Grandis JR: Gene therapy for the treatment of oral squamous cell carcinoma. J Dent Res 82: 11-16, 2003.

7. Lo WY, Tsai MH, Tsai Y, Hua CH, Tsai FJ, Huang SY, Tsai $\mathrm{CH}$ and Lai CC: Identification of over-expressed proteins in oral squamous cell carcinoma (OSCC) patients by clinical proteomic analysis. Clin Chim Acta 376: 101-107, 2007.

8. Zygogianni AG, Kyrgias G, Karakitsos P, Psyrri A, Kouvaris J, Kelekis N and Kouloulias V: Oral squamous cell cancer: Early detection and the role of alcohol and smoking. Head Neck Oncol 3: 2, 2011.

9. Taniguchi H, Manenti S, Suzuki M and Titani K: Myristoylated alanine-rich $\mathrm{C}$ kinase substrate (MARCKS), a major protein kinase $\mathrm{C}$ substrate, is an in vivo substrate of proline-directed protein kinase(s). A mass spectroscopic analysis of the post-translational modifications. J Biol Chem 269: 18299-18302, 1994.

10. Brooks G: The role of $80 \mathrm{~K} / \mathrm{MARCKS}$, a specific substrate of protein kinase $\mathrm{C}$, in cell growth and tumour progression. Pigment Cell Res 7: 451-457, 1994.

11. Jarboe JS, Anderson JC, Duarte CW, Mehta T, Nowsheen S, Hicks PH, Whitley AC, Rohrbach TD, McCubrey RO, Chiu S, et al: MARCKS regulates growth and radiation sensitivity and is a novel prognostic factor for glioma. Clin Cancer Res 18: 3030-3041, 2012.

12. Ulbrich C, Pietsch J, Grosse J, Wehland M, Schulz H, Saar K, Hübner N, Hauslage J, Hemmersbach R, Braun M, et al: Differential gene regulation under altered gravity conditions in follicular thyroid cancer cells: Relationship between the extracellular matrix and the cytoskeleton. Cell Physiol Biochem 28: 185-198, 2011.
13. Rombouts K, Carloni V, Mello T, Omenetti S, Galastri S, Madiai S, Galli A and Pinzani M: Myristoylated alanine-rich protein kinase C substrate (MARCKS) expression modulates the metastatic phenotype in human and murine colon carcinoma in vitro and in vivo. Cancer Lett 333: 244-252, 2013.

14. Chen X and Rotenberg SA: PhosphoMARCKS drives motility of mouse melanoma cells. Cell Signal 22: 1097-1103, 2010.

15. Micallef J, Taccone M, Mukherjee J, Croul S, Busby J, Moran MF and Guha A: Epidermal growth factor receptor variant III-induced glioma invasion is mediated through myristoylated alanine-rich protein kinase $\mathrm{C}$ substrate overexpression. Cancer Res 69: 7548-7556, 2009.

16. Techasen A, Loilome W, Namwat N, Takahashi E, Sugihara E, Puapairoj A, Miwa M, Saya H and Yongvanit P: Myristoylated alanine-rich $\mathrm{C}$ kinase substrate phosphorylation promotes cholangiocarcinoma cell migration and metastasis via the protein kinase C-dependent pathway. Cancer Sci 101: 658-665, 2010.

17. Livak KJ and Schmittgen TD: Analysis of relative gene expression data using real-time quantitative PCR and the 2(-Delta Delta C(T)) method. Methods 25: 402-408, 2001.

18. Glaser M, Wanaski S, Buser CA, Boguslavsky V, Rashidzada W, Morris A, Rebecchi M, Scarlata SF, Runnels LW, Prestwich GD, et al: Myristoylated alanine-rich C kinase substrate (MARCKS) produces reversible inhibition of phospholipase $C$ by sequestering phosphatidylinositol 4,5-bisphosphate in lateral domains. J Biol Chem 271: 26187-22693, 1996.

19. Ueno E and Rosenberg P: Beta-Bungarotoxin blocks phorbol ester-stimulated phosphorylation of MARCKS, GAP-43 and synapsin I in rat brain synaptosomes. Toxicon 33: 747-762, 1995.

20. Woo JH, Wilsbach K, Nordin A, Lorincz A and Gabrielson E: Increased expression of MARCKS in cancer cells represents a potential target for treatment. Cancer Res 64, 2004.

21. Manai M, Thomassin-Piana J, Gamoudi A, Finetti P, Lopez M, Eghozzi R, Ayadi S, Lamine OB, Manai M, Rahal K, et al: MARCKS protein overexpression in inflammatory breast cancer. Oncotarget 8: 6246-6257, 2017.

22. Hanada S, Kakehashi A, Nishiyama N, Wei M, Yamano S, Chung K, Komatsu H, Inoue H, Suehiro S and Wanibuchi H: Myristoylated alanine-rich C-kinase substrate as a prognostic biomarker in human primary lung squamous cell carcinoma. Cancer Biomark 13: 289-298, 2013.

23. Chen CH, Fong LWR, Yu E, Wu R, Trott JF and Weiss RH: Upregulation of MARCKS in kidney cancer and its potential as a therapeutic target. Oncogene 36: 3588-3598, 2017.

24. Hers I, Vincent EE and Tavaré JM: Akt signalling in health and disease. Cell Signal 10: 1515-1527, 2011.

25. Fruman DA and Rommel C: PI3K and cancer: Lessons, challenges and opportunities. Nat Rev Drug Discov 13: 140-156, 2014.

26. Carnero A, Blanco-Aparicio C, Renner O, Link W and Leal JF: The PTEN/PI3K/AKT signalling pathway in cancer, therapeutic implications. Curr Cancer Drug Targets 8: 187-198, 2008.

27. Ziemba BP, Burke JE, Masson G, Williams RL and Falke JJ: Regulation of PI3K by PKC and MARCKS: Single-molecule analysis of a reconstituted signaling pathway. Biophys J 110: 1811-1825, 2016.

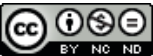

This work is licensed under a Creative Commons Attribution-NonCommercial-NoDerivatives 4.0 International (CC BY-NC-ND 4.0) License. 